\title{
Is there a standard procedure for assessing and providing assistive devices for people with neuro-disabling conditions in United Kingdom? A nation-wide survey
}

\author{
L. Tedesco Triccas ${ }^{\text {a, b, }{ }^{*}, \text { B. McLening }}{ }^{\text {c }}$, W. Hendrie ${ }^{\text {d }}$, G. Peryer ${ }^{\text {a }}$ \\ a School of Health Sciences, University of East Anglia, Norwich, NR4 7TJ, United Kingdom \\ ${ }^{\mathrm{b}}$ Department of Rehabilitation Sciences, KU Leuven, Tervuursevest 101, Heverlee, 3001, Belgium \\ ${ }^{\mathrm{c}}$ Norfolk Community Health and Care NHS Trust, Norfolk, United Kingdom \\ ${ }^{\mathrm{d}}$ MS Therapy Centre, 5 Hurricane Way, Norwich NR6 6EZ, United Kingdom
}

\section{A R T I C L E I N F O}

\section{Article history:}

Received 26 March 2018

Received in revised form

27 June 2018

Accepted 1 August 2018

\section{Keywords:}

Assistive technology

Stroke

Multiple sclerosis

Parkinson's disease

Guidelines

\begin{abstract}
A B S T R A C T
Background: Assistive devices are currently provided to people with neuro-disabling conditions to promote or maintain independence in activities of daily living. However, it is unclear whether assessment procedures performed by health care professionals to guide the provision of assistive devices are standardized.

Objective: To explore the assessment and service-delivery processes of assistive devices for people with multiple sclerosis, cerebrovascular disease and Parkinson's disease experiencing physical disability by health care professionals in the United Kingdom.

Methods: A survey was conducted among UK health care professionals working with people with neurodisabling conditions. Descriptive and content analyses were used to code survey data.

Results: In total, 231 health care professionals completed the survey: 93 occupational therapists, 136 physiotherapists and 2 assistant practitioners. Less than half of the respondents (46\%) reported use of local, national, or combined guidelines when assessing a service user's suitability or need for assistive devices. When guidelines were used, they were not consistent and not specifically for assistive devices. The respondents stated that when users were allocated small and portable assistive devices, they were supplied within four weeks. This period increased for large equipment, major home adaptions or if external specialist services and/or funding was needed.

Conclusions: Standardized operating procedures for assistive device provision are not being carried out within the UK. Variable access to assistive devices supplied by the state indicates inequity across regions. Future research should explore potential benefits of developing standardized assessment procedures for the provision of assistive devices and devise methods to reduce current variability in service delivery.
\end{abstract}

(c) 2018 Elsevier Inc. All rights reserved.

\section{Introduction}

In the Global Burden of Disease study, people with severe impairments resulting from stroke, Parkinson's disease and multiple sclerosis have been associated with higher disability in comparison with other neurological conditions. ${ }^{1}$ These neuro-disabling conditions are also associated with neurological symptoms such as

\footnotetext{
* Corresponding author. Faculty of Kinesiology and Rehabilitation Sciences, Department of Rehabilitation Sciences, KU Leuven, Tervuursevest 101, box 1500, B3001, Heverlee, Belgium.

E-mail address: lisa.tedescotriccas@kuleuven.be (L. Tedesco Triccas).
}

tremor, muscle weakness, and balance impairments which can affect all aspects of the International Classification of Functioning, Disability and Health scale. ${ }^{2}$ These symptoms can contribute to limitations and severe impairments when engaging in functional activities of daily living (ADLs), thereby restricting participation and increasing dependence on carers. ${ }^{3}$

People experiencing difficulties with ADLs are commonly referred to rehabilitation services, including physiotherapy and occupational therapy, to ameliorate functional limitations. ${ }^{4}$ In many cases this involves a needs assessment and the provision of certain assistive technologies. The World Health Organization defines assistive devices and technologies as: "those whose primary purpose is to maintain or improve an individual's functioning and 
independence to facilitate participation and enhance overall wellbeing". ${ }^{5}$ Assistive devices aim to address the negative impact of a neuro-disabling condition. According to the World Report on Disability, assistive devices promote independence and social participation. ${ }^{6}$ Assistive devices such as grab rails, bath aids, hoists, ramps and wheelchairs aim to promote independence during mobility, activities and personal care, increase safety, and decrease burden on carers. ${ }^{7-11}$

Limitations experienced by people with neuro-disabling conditions can increase over time and therefore assistive device provision may require periodic review. A focus group conducted prior to this study identified that people with multiple sclerosis with moderate to severe disability experienced a range of barriers to the assessment and provision of assistive devices by the National Health Service (NHS). These included:long waiting times, a lack of an initial and follow-up assessment and a mismatch of the devices provided according to the individual's needs. In some instances, this led to early abandonment. Early abandonment is a wellrecognized issue ${ }^{12,13}$ and one third of assistive technologies provided are subsequently abandoned. ${ }^{14,15}$

It is crucial to assess and provide assistive technology in a timely manner and ensure that users are central to the decision-making process. ${ }^{16}$ In the current scientific literature, there are two main models for the selection of and advisory process for assistive devices or technology. The Human Activity Assistive Technology (HAAT) model addresses personal factors including characteristics and symptoms of the condition, as well as contextual factors, including the type of social support and current state of finances of the person being assessed for assistive technology. ${ }^{17}$ This model puts emphasis on the evaluation of the assistive devices selection process but has not been tested for validity, does not include tools for assessment and does not explore to what extent the use of assistive devices continues after they are provided to disabled people. ${ }^{18}$ The second model, called The Matching Person and Technology Model (MPT), facilitates the selection of the most suitable assistive technology in relation the consumer's perspectives and abilities, the specifics of the assistive technology and the individual's environmental conditions. It has been found to be reliable in the United States of America, Canada, Australia and Ireland. ${ }^{19}$ Within the NHS and Social Services, patients with neuro-disabling conditions are usually assessed for their needs for assistive devices based on their symptoms and likely disease progression, current abilities and environment by health care professionals. However, currently unknown to what extent the HAAT, MPT or similar guidelines, are used or preferred by health care professionals in the United Kingdom (UK).

The objectives of this study, therefore, were to explore current assessment practices by UK healthcare professionals and subsequent provision and review of assistive devices, for people with multiple sclerosis, stroke and Parkinson's disease experiencing physical disability.

\section{Method}

Ethical approval to conduct this research was obtained from the School of Health Sciences Ethics Committee, University of East Anglia (reference: 20152016-1).

\section{Target sample}

A convenience sample of health care professionals with an experience of assistive devices provision was approached and asked to participate. Participants needed to be: (i) $>18$ years old; (ii) currently working or having previously worked in in-patient or community settings; (iii) currently or previously responsible for undertaking assessment for the need and provision of assistive devices for service users.

\section{Survey development and pre-testing}

An open survey was developed by a team of researchers at the School of Health Sciences, University of East Anglia (UEA) and an occupational therapist from the Norfolk and Community Health and Care NHS Trust. In line with the research question, items were developed based on measuring the attitudes and opinions of health care professionals about the assessment and provision of assistive devices. ${ }^{20}$ These were developed from the issues about the long waiting times for provision and assessment process raised by the participants during the focus group. The survey combined a combination of open- and close-ended questions. In some instances, Likert-scales were used for close-ended questions. The survey was made available online using Survey Monkey ${ }^{\mathbb{B}}$. The e-survey was piloted by two researchers and two health care professionals working with people with neuro-disabling conditions.

The final version contained 13 items that focused on: the participants' level of experience, the type of assistive devices they provide, the duration between assessment and provision, whether they follow clinical guidelines when conducting assessments and whether a follow-up consultation takes place. The final version of the survey is presented in the Appendix.

\section{Recruitment and data collection}

As this was a scoping exercise, a snowball sampling was carried out. ${ }^{21}$ Health care professionals were identified and recruited via the research leads of: (1) Royal College of Occupational Therapists and (2) Chartered Society of Physiotherapists. In addition, participants were recruited from the following specialist interest groups in neurology: (3) Royal College of Occupational Therapist Specialist Section- Neurological Practice and (4) Association of Chartered Physiotherapists in Neurology. An email invitation was sent to the identified members of the organizations with a cover letter which introduced the study and, contained a link to the participant information and the e-survey. Data were collected from October 2015 until February 2016. Participants were able to review and change their answers on the e-survey. A unique user identifier was provided to each participant to avoid duplicate survey entry.

\section{Data analysis}

Following collection, nominal and ordinal data were downloaded from Survey Monkey ${ }^{\circledR}$ in an Excel format then imported into the Statistical Package for the Social Sciences (SPSS Version 19.0, IBM Corp, Armonk NY). Descriptive statistics were calculated from all completed entries. Likert scale responses were analyzed using percentages calculated by the number of respondents from the total sample that responded to each item of the scale. Pivot table analysis was used as a sub-group analysis in Excel to explore any trends within UK counties and years of experience of health care professionals who use guidelines. ${ }^{22}$ Responses to open-ended questions were analyzed using inductive content analysis, ${ }^{23,24}$ which involved classification and reduction of data into contextual elements. ${ }^{25}$ This process identified recurring codes by LTT, which were then discussed and modified accordingly by LTT and BM.

\section{Results}

In total, 231 health care professionals completed the survey: 93/ 231 (40\%) occupational therapists, 136/231 (59\%) physiotherapists and $2 / 231(1 \%)$ assistant practitioners. The majority of the 
participants $217 / 231$ (94\%) worked within the NHS and 14/231 (6\%) worked within the Social Services. From the respondents, 214 (93\%) worked in England, 9 (4\%) worked in Scotland, 7 (3\%) worked in Wales and $1(<1 \%)$ worked in Northern Ireland. The work setting of participants included: in-patient 58/231 (25\%), out-patient 96/231 (42\%), specialist neurology $73 / 231$ (32\%) and general 4 (2\%). The mean level of experience was 11.3(SD: 7.95) years.

Following assessment, health care professionals provided mainly aids for mobility $216 / 231$ (94\%) and aids for moving and handling 202/231 (87\%) (Fig. 1). In addition, 58/231 (25\%) provided other devices including: bathing and seating equipment, splinting, orthotics, rehabilitation health technologies such as Functional Electrical Stimulation, environmental control systems and monitoring systems such as telecare, fall and bed sensors.

More than half of the respondents 124/231 (54\%) stated that they do not follow any guidelines when assessing for assistive devices. From the participants who use guidelines, 70/104 (67\%) use local guidelines, 18/104 (2\%) use national guidelines, and 16/104 use both local and national guidelines. Data from the open questions about the type of guidelines used by the respondents were the following: local included (i) NHS Trust specific, and national included (ii) OT specifically based such as Royal College of Occupational Therapists (RCOT practice guidelines, 2017) ${ }^{26}$; (iii) $\mathrm{Na}$ tional Institute for Health and Care Excellence for multiple sclerosis and Functional Electrical Stimulation ${ }^{27,28}$; (iv) UK company-based guidelines that provides assistive devices (NRS Healthcare) and (v) National Service Framework for Long Term Conditions, and Care Act guidelines ${ }^{29}$. From pivot table analysis, there were not any trends within UK counties and years of experience of health care professionals concerning those who use guidelines compared to those who do not.

Health care professionals stated that people with neurodisabling conditions were assessed for assistive devices: immediately 56/230 (24\%), within a week 49/230 (21\%), between 1 and 4 weeks 63/230 (27\%) or between 1 and 6 months 44/230 (19\%) (Fig. 2). Assessments for assistive devices were reported to be conducted face to face $205 / 231$ (89\%), purely via the telephone 1 / $231(<1 \%)$, or both face to face and via the telephone $25 / 231(10.8 \%)$. Once the equipment is installed or provided, 166/231 (72\%) carry out a follow-up consultation. Following assessment, assistive devices were provided: immediately $17 / 231$ (7.4\%), within a week $101 / 231$ (43.7\%), between 1 and 4 weeks 70/231 (30.3\%), between 1 and 6 months $22 / 231$ (9.5\%), between 6 and 12 months $1 / 231$ $(0.4 \%)$, more than a year $1 / 231(0.4 \%)$ and some respondents did not know 19/231 (8.2\%) (Fig. 3).

The main reasons for the long waiting time were: (1) type and size of item-larger items took longer, (2) dependent on an external specialist service and (3) dependent on funding and the financial situation of the person with the neuro-disabling condition.

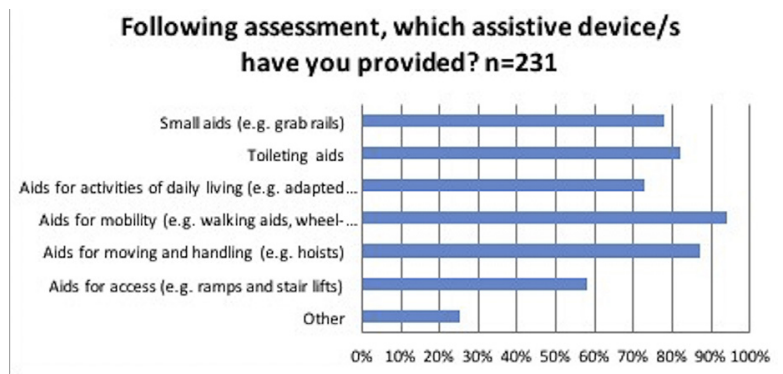

Fig. 1. Type of assistive devices (\%) provided to people with neuro-disabling conditions after assessment by health care professionals.

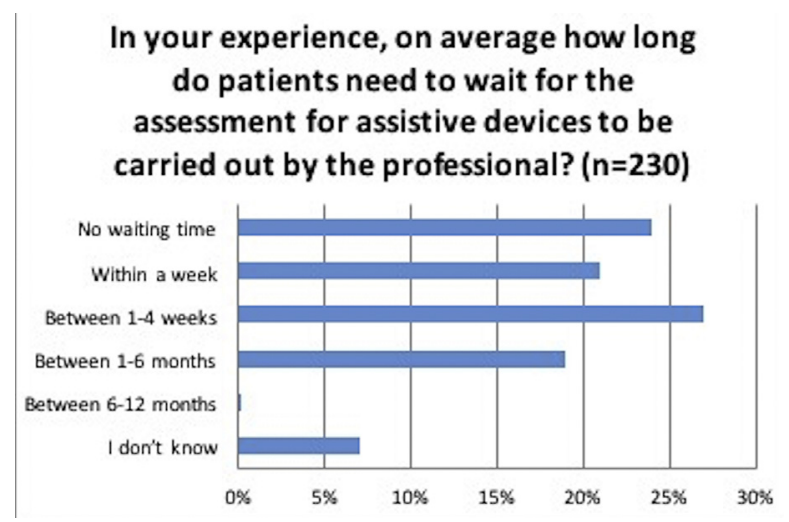

Fig. 2. Showing percentage waiting time by person with neuro-disabling condition for the assessment of assistive devices.

Type and size of item-larger items took longer

The majority of the respondents agreed that if the assistive device was readily available from a store, then that item could be provided to the user within $24 \mathrm{~h}$. It was stated that 'major' compared to 'minor' adaptations to the house, such as installing a stair lift, could take up to six months.

\section{Dependent on an external specialist service}

Respondents identified that major adaptations and equipment required a lengthy assessment and referral process to an external organization or company. This involved evaluating prospective risk, such as an increase in falls and then obtaining a completed consent from the user for an assistive device. Health care professionals stated that length of waiting time seemed to depend on whether or not the local social services accepted the assessment from the NHS. In addition, installation of equipment depended on the availability of technicians. The two main organizations, NHS and Social Services, seemed to face challenges when working together. Health care professionals working within the NHS stated that major adaptations "are a huge issue, as this is social service-led" [P228].

\section{Dependent on funding and financial situation of the user}

Waiting time also depended on two issues: funding bodies and the financial situation of the user. Small equipment, such as hand rails and walking sticks, were funded by the NHS or social services using 'community equipment loan stores'. However, more expensive equipment, such as wheelchairs and bath lifts, required an

\section{Post-assessment, generally how long do patients wait for the equipment to be provided? $(n=230)$}

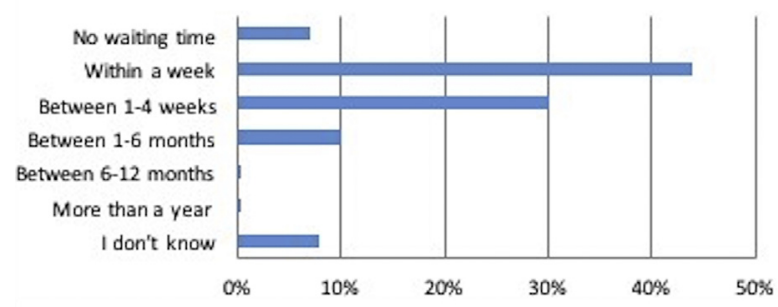

Fig. 3. Showing percentage waiting time by person with neuro-disabling condition for the provision of assistive devices. 
application for external funding. The latter had to "be approved by a panel and then sourced" [P159]. If the equipment was self-funded by the patient, the waiting time was less.

\section{Discussion}

This study has given rise to new knowledge about current routine practice for the assessment and provision of assistive devices for people with neuro-disabling conditions. The results from the survey showed that less than half of the respondents used guidelines during the assessment process. It appears that a standardized practice for assistive device provision is not being applied routinely within the UK. Delays between assessment and provision of assistive devices depended on the size of the equipment, the scale of adaptations required for installation, coordination of multiple service providers and source of funding.

Generally, there is not a tendency for standardized guidelines to be used during assessment and provision of assistive devices in the UK. In a similar survey, only $20 \%$ of health care professionals used guidance with users when measuring home assistive devices. ${ }^{30}$ Clinical guidelines should be based on evidence-based recommendations. ${ }^{31}$ Therefore, one reason for the lack of use could be due to the limited high-quality evidence-based procedures for assistive device selection in neurological settings. ${ }^{19}$ Lack of alignment of protocol use could be the reasons for the delay in the provision of assistive devices and home adaptations. ${ }^{31}$ These delays could cause patient and carer strain and increase disease symptoms and functional limitations. ${ }^{32-34}$

In the present study, it was reported that more than a quarter of the health care professionals do not carry out a follow-up assessment of the assistive devices provided. This could be a contributory factor as to why $28.6-50 \%$ of mobility assistive devices are currently abandoned in the stroke population. ${ }^{35}$ Thus, ensuring that the assistive device fits the lifestyle and needs of the patient should be compulsory and follow-up should be addressed when developing future guidelines which could decrease abandonment. ${ }^{14}$ This could limit waste of funds spent by national health systems.

This is the first survey exploring the topic of assistive device provision with a large sample of health care professionals in the UK. However, limitations should also be acknowledged. Recruitment of a large number of health care professionals from social services would have improved the sample representativeness from both sectors. Also, due to the use of a snowball sampling technique, we did not know the exact number of potential participants asked to complete the survey. Additionally, respondents were not selected in a random order and, therefore, the open question responses could have been biased towards expressing views of people who had a negative experience from assistive device services. The respondents worked with different neuro-disabling conditions and, therefore, the results may not be generalizable to a particular condition such as stroke. Finally, in order to adhere to the minimal time needed for survey completion, the age and type of gender were not included as items in the survey and, therefore, sociodemographic information about the respondents is minimal.

\section{Future research}

Clinical guidelines have the potential to improve quality of care and improve outcome. ${ }^{36,37}$ Using standardized clinical guidelines has implications for increased efficiency of assistive device provision and optimizing value of money in health and social care provision. ${ }^{38}$ When an assistive device implementation and evaluation protocol was implemented for 54 people with multiple sclerosis in a samll study Italy, this resulted in a significant decrease in device abandonment. $^{12}$
The next step for future research should explore the development and implementation of a user-led, standardized guideline for assistive devices. This should involve input from a large sample of people with neuro-disabling conditions as well as health care professionals who have expertise in the use and prescription of assistive devices. Guideline development expertise and endorsement for the final document should also be sought from the professional bodies who should be involved in the project from the outset. As a result, it would be then important to evaluate the level of abandonment and satisfaction of the client and carers once implemented within the UK. ${ }^{39}$ Due to the fact that there is a broad range of assistive devices, specific guidelines for the sub-groups of assistive devices (such as walking aids, manual handling equipment) should also be considered, to promote a client-centered approach. Such guidelines should incorporate the evaluation of the needs and suitability of the personal and contextual individual factors. ${ }^{11}$ Additionally, appropriate outcome measures such as the Quebec User Evaluation of Satisfaction with Assistive Technology, ${ }^{40}$ the Carer Asssistive Technology Outcome Measure, ${ }^{41}$ or the Psychsocial Impact of Assistive Devices Scales ${ }^{42}$ should be used as immediate and long-term follow-up assessments of effectiveness of guideline implementation. ${ }^{43-45}$

\section{Conclusion}

This study has provided some knowledge of the current practice used for the assessment and provision of assistive devices for people with neuro-disabling conditions by health care professionals in UK. The delay in the provision of assistive devices depended on the size of the item, the size of the adaptations needed for the installation of assistive devices, lack of coordination between services and funding. Results showed that health care professionals do not follow a consistent standardized procedure for service delivery of assistive devices. Therefore, future research should explore the development and implementation of standardized guidelines to be used by all Trusts and services in the UK. This could potentially enable effective assessment of suitability and provision of assistive devices and ensure that people with neurodisabling conditions get the equipment they need to live as independently as possible.

\section{Conflicts of interest}

None declared.

\section{Financial support}

The research work disclosed in this publication is partially funded by the REACH HIGH Scholars Programme - Post-Doctoral Grants. The project is part-financed by the European Union, Operational Programme II - Cohesion Policy 2014-2020 Investing in human capital to create more opportunities and promote the wellbeing of society -European Social Fund.

\section{Acknowledgements}

We would like to thank the participants with multiple sclerosis and their carers who took part in the focus group prior to the survey and also the respondents for taking time to complete the survey.

\section{Appendix A. Supplementary data}

Supplementary data related to this article can be found at https://doi.org/10.1016/j.dhjo.2018.08.003. 


\section{References}

1. Stovner LJ, Hoff JM, Svalheim S, Gilhus NE. Neurological disorders in the global burden of disease 2010 study. Acta Neurol Scand. 2014;129(s198):1-6.

2. World Health Organization. International Classification of Functioning, Disability and Health: ICF. World Health Organization; 2001.

3. Ben Ari E, Johansson S, Ytterberg C, Bergström J, von Koch L. How are cognitive impairment, fatigue and signs of depression related to participation in daily life among persons with multiple sclerosis? Disabil Rehabil. 2014;36(23): 2012-2018.

4. Salminen AL, Kanelisto KJ, Karhula ME. What components of rehabilitation are helpful from the perspective of individuals with multiple sclerosis? Disabil Rehabil. 2014;36(23):1983-1989.

5. World Health Organisation. Disability. http://www.who.int/disabilities/ technology/en/; 2018. Accessed March 15, 2018.

6. Shakespeare T, Officer A. World report on disability. Disabil Rehabil. 2011;33(17-18):1491.

7. Anderson WL, Wiener JM. The impact of assistive technologies on formal and informal home care. The Gerontologist. 2013;165.

8. Greenhalgh T, Wherton J, Sugarhood P, Hinder S, Procter R, Stones R. What matters to older people with assisted living needs? A phenomenological analysis of the use and non-use of telehealth and telecare. Soc Sci Med. 2013;93:86-94.

9. Mortenson WB, Demers L, Fuhrer MJ, Jutai JW, Lenker J, DeRuyter F. Effects of an assistive technology intervention on older adults with disabilities and their informal caregivers: an exploratory randomized controlled trial. Am J Phys Med Rehabil. 2013;4:297-306.

10. Scherer MJ, Craddock G, Mackeogh T. The relationship of personal factors and subjective well-being to the use of assistive technology devices. Disabil Rehabil. 2011;33(10):811-817.

11. Souza A, Kelleher A, Cooper R, et al. Multiple sclerosis and mobility-related assistive technology: systematic review of literature. I Rehabil Res Dev. 2010;47(3):213-223.

12. Verza R, Carvalho ML, Battaglia MA, Uccelli MM. An interdisciplinary approach to evaluating the need for assistive technology reduces equipment abandonment. Mult Scler J. 2006;12(1):88-93.

13. Wessels R, Dijcks B, Soede M, Gelderblom GJ, De Witte L. Non-use of provided assistive technology devices, a literature overview. Technol Disabil. 2003:1:15(4):231-238.

14. Sugawara AT, Ramos VD, Alfieri FM, Battistella LR. Abandonment of assistive products: assessing abandonment levels and factors that impact on it. Disabil Rehabil Assist Technol. 2018:1-8.

15. Federici S, Meloni F, Borsci S. The abandonment of assistive technology in Italy: a survey of National Health Service users. Eur J Phys Rehabil Med. 2016;52(4): $516-526$.

16. Desmond D, Layton N, Bentley J, et al. Assistive technology and people: a position paper from the first global research, innovation and education on assistive technology (GREAT) summit. Disabil Rehabil Assist Technol. 2018:1-8.

17. Cook AM, Hussey SM. Assistive Technologies: Principles and Practice. St.Louis, MO: Mosby, Inc; 1995.

18. Squires LA, Williams N, Morrison VL. Matching and accepting assistive technology in multiple sclerosis: a focus group study with people with multiple sclerosis, carers and occupational therapists. J Health Psychol. 2016;15 1359105316677293.

19. Bernd T, Van Der Pijl D, De Witte LP. Existing models and instruments for the selection of assistive technology in rehabilitation practice. Scand J Occup Ther 2009:16(3):146-158.

20. Bowling A. Research Methods in Health: Investigating Health and Health Services. UK: McGraw-Hill Education; 2014

21. Biernacki P, Waldorf D. Snowball sampling: problems and techniques of chain referral sampling. Socio Meth Res. 1981;10(2):141-163.

22. Stolte C, Tang D, Hanrahan P. Polaris: a system for query, analysis, and visualization of multidimensional relational databases. IEEE Trans Visual Comput Graph. 2002;8(1):52-65.

23. Elo S, Kyngäs $\mathrm{H}$. The qualitative content analysis process. J Adv Nurs. 2008;62(1):107-115.

24. Weber RP. Basic Content analysis (No. 49). Sage; 1990.

25. Hsieh HF, Shannon SE. Three approaches to qualitative content analysis. Qual Health Res. 2005;15(9):1277-1288.

26. RCOT Practice Guidelines; 2017. https://www.rcot.co.uk/practice-resources/ rcot-practice-guidelines. Accessed February 17, 2018.

27. Multiple Sclerosis in Adults: Management. National Institute for Health Care and Excellence; 2014. https://www.nice.org.uk/guidance/cg186. Accessed February 17, 2018.

28. Functional Electrical Stimulation for Drop Foot of Central Neurological Origin. National Institute for Health Care and Excellence; 2009. https://www.nice.org. uk/guidance/ipg278. Accessed February 17, 2018.

29. Guidance National Service Framework: Long Term Conditions; 2015. https:// www.gov.uk/government/publications/quality-standards-for-supportingpeople-with-long-term-conditions. Accessed February 18, 2018.

30. Atwal A, Mcintyre A, Spiliotopoulou G, Money A, Paraskevopulos I. How are service users instructed to measure home furniture for provision of minor assistive devices? Disabil Rehabil Assist Technol. 2017;12(2):153-159.

31. Rycroft-Malone J. Formal consensus: the development of a national clinical guideline. BMJ Qual Saf. 2001;10(4):238-244.

32. Henschke C. Provision and financing of assistive technology devices in Germany: a bureaucratic odyssey? The case of amyotrophic lateral sclerosis and Duchenne muscular dystrophy. Health Pol. 2012;105(2):176-184.

33. Wessels R, Dijcks B, Soede M, Gelderblom GJ, De Witte L. Non-use of provided assistive technology devices, a literature overview. Technol Disabil. $2003 ; 1 ; 15(4): 231-238$.

34. Jedeloo S, Witte LD, Linssen BAJ, Schrijvers AJP. Client satisfaction with service delivery of assistive technology for outdoor mobility. Disabil Rehabil. 2002;24(10):550-557.

35. Safaz I, Türk H, Yașar E, Alaca R, Tok F, TUğCU I. Use and abandonment rates of assistive devices/orthoses in patients with stroke. Gulhane Med J. 2015·57(2).

36. Woolf SH, Grol R, Hutchinson A, Eccles M, Grimshaw J. Potential benefits, limitations, and harms of clinical guidelines. Bmj. 1999;318(7182):527-530.

37. Grimshaw JM, Russell IT. Effect of clinical guidelines on medical practice: a systematic review of rigorous evaluations. Lancet. 1993:342(8883):1317-1322.

38. Shapiro DW, Lasker RD, Bindman AB, Lee PR. Containing costs while improving quality of care: the role of profiling and practice guidelines. Annu Rev Publ Health. 1993:14:219-241.

39. Larsson Ranada Å, Lidström H. Satisfaction with assistive technology device in relation to the service delivery process-a systematic review. Assist Technol. 2017:1-16.

40. Demers L, Weiss-Lambrou R, Ska B. The Quebec user evaluation of satisfaction with assistive technology (QUEST 2.0): an overview and recent progress. Technol Disabil. 2002;14(3):101-105.

41. Mortenson WB, Demers L, Fuhrer MJ, Jutai JW, Lenker J, DeRuyter F. Development and preliminary evaluation of the caregiver assistive technology outcome measure. J Rehabil Med. 2015;47(5):412-418.

42. Jutai J, Day H. Psychosocial impact of assistive devices scale (PIADS). Technol Disabil. 2002;14(3):107-111.

43. Scherer M, Jutai J, Fuhrer M, Demers L, Deruyter F. A framework for modelling the selection of assistive technology devices (ATDs). Disabil Rehabil Assist Technol. 2007;2(1):1-8.

44. Cook AM, Hussey SM. Control Interfaces for Assistive Technology. Assistive Technologies: Principles and Practice. second ed. St. Louis: Mosby, Inc; 2002: $212-254$

45. Smith RO, Scherer MJ. Where Are We Headed with Assistive Technology Outcomes. RESNA Resource Guide for Assistive Technology Outcomes: Measurement Tools. first ed. 1998 